\title{
Patient-centred services
}

\author{
J. A. Spall \\ Chair, Children's Heart Federation, London, UK
}

I T IS INTERESTING AND TIMELY THAT THIS FASCICLE OF the Journal contains three articles based upon the views of the patient, or the parents of the patient. Timely because the whole question of involvement of patients and their parents, and the development of patient-centred services, is gathering momentum, not least in paediatric cardiology.

Some of the reasons for this lie in wider social changes over the past twenty years, others in more immediate events. The rise of consumerism has, for differing reasons, been on the agenda of both the main political parties of the United Kingdom since the early 1980s, one first espousing the consumer as a free enterprise purchaser of privatised services, with the other later seeing the consumer as a weapon in tackling the perceived ills of producer-dominated public services. In both cases, there is an emphasis on the rights of the individual, and an attempt to shift power, thus creating more balance between the corporate and consumer sections of society.

An expansion of the "middle classes" - more articulate, more aware, better educated and often having the skills to access and assess information - has meant that the aura of the more traditional professions, based on an exclusive knowledge base, has lessened.

Information is now globally available via the Internet. This provides patients with access to treatments, practices, and standards in other developed countries. For those in the United Kingdom, this permits comparisons to be made of what is available in the National Health Service. This is sometimes to its detriment, suffering as it has been from many years of underfunding.

A strident campaigning, and sensation-seeking, press have been eager to seek out real and perceived ills, usually failing to draw too fine a distinction

Correspondence to: Mr J. A. Spall, Chair, Children's Heart Federation, 52 Kennington Oval, London SE11 5SW, UK. Tel: 0207820 8517; Fax: 020 7735 8718; E-mail: chf@dircon.co.uk

Accepted for publication 15 November 2002 between the two. Alongside this, there has developed a litigious and compensation-seeking culture. This period has also been characterised by a general lessening of respect for authority, and figures of authority. These trends are, of course, not exclusive to the United Kingdom, and elements of them can be found in all developed countries.

Within the United Kingdom, nonetheless, the Government produced, in 2000, its National Plan for the National Health Service. ${ }^{1}$ This document emphasised moves towards a service centred on the patient, or in the case of many of those with congenitally malformed hearts, the parents acting on behalf of the patient. This would probably have trundled along, as these sorts of grandiose documents do, having some impact but probably not changing much fundamentally, but for the events at the Bristol Royal Infirmary that triggered the Kennedy Inquiry and Report. ${ }^{2}$ At about the same time, the issue of retention of organs at Alder Hey, in Liverpool, ${ }^{3}$ and the inquiry into standards of treatment, particularly for those with Down's syndrome, at the Royal Brompton Hospital in London, ${ }^{4}$ were both overshadowed by the Kennedy Inquiry, but shone further light into dark corners of medical practice.

The first part of the Kennedy Report ${ }^{2}$ described a catalogue of errors, ranging from the machinations which allowed Bristol to be designated a "centre of excellence," even though it did not meet the criteria so to be, to activities at the level of local management and clinical care. In the second part, Kennedy and his colleagues moved on to propose a new blueprint for the National Health Service, which included the proposition that "patients must be at the centre of the NHS and the patients' perspective must be included in the policy, planning and delivery of services at every level".

In its response, ${ }^{5}$ the Department of Health said, "by helping patients make choices we will ensure that hospitals respond more directly to the needs and wishes of patients." The Department also endorsed 
the concept proposed by Kennedy and his colleagues of "partnership between the patient and health professionals in the exchange and provision of information in the way forward to developing a culture of openness and honesty".

Fine words and soaring ambitions - but how were these to be turned from rhetoric to real change on the ground? It was at least good news to learn that the Department of Health was to set up a Paediatric and Congenital Cardiac Services Review Group to assess the likely need for services looking ten to twenty years ahead; to review current service provision, activity and outcome; consider likely changes in organisation and delivery; taking into account the various reports, views of patients and parents; and recommend national standards. Even more, that the group would include a few user representatives, of whom I was one, as chair of the Children's Heart Federation, the national umbrella charity for parent support groups.

Support groups for families of children with congenital heart disease first appeared in the 1970s, and increased in number through the 1980s and 1990s. Two types of group were in evidence - those geographically based relating to one or several paediatric cardiac units, and national groups supporting families with a child with a particular cardiac condition.

For some of these groups, the initial motivation was to raise funds to provide much needed equipment for the local unit. Many later moved also into facilitating support services for the families. For others, particularly the national groups, support was the main motivation from the outset. In either case, campaigning for change, or monitoring what went on in the units, was not seen as an appropriate activity. Indeed, groups were fiercely loyal about their own unit, and the doctors and other staff working there.

Bristol, therefore, came as a big shock to many groups. Some reacted by largely ignoring it. Some took the view that it couldn't happen at their unit. In a few cases, particularly among the national groups supporting one condition, it confirmed the need to take a more pro-active and campaigning role. This relative vacuum led to the establishment of local action groups of parents, which then campaigned with skill, passion and persistence, arguing that actions at the relevant units should be brought to public scrutiny, errors admitted, and changes made. In so doing, they advanced the idea of a campaigning role generally for support groups, and particularly for the national umbrella charity, the Children's Heart Federation. While none of these action groups were wholly satisfied with the outcomes of the various reports, there has been a general welcome from user support organisations for the changes proposed.

What progress has so far been achieved? There have been some modest advances. The review group examining paediatric cardiac services on behalf of the Department of Health has made real attempts to seek user opinion. It has consulted with all support groups, via meetings brokered by the Children's Heart Federation, and face-to-face discussions with representatives of the patients and their parents at every paediatric cardiac unit. Similarly, representatives of parents are involved in the forum implementing changes proposed in the Evans Report ${ }^{4}$ that emerged from the inquiry into the happenings at Royal Brompton Hospital.

A third encouraging development has been increased co-operation, and joint activity, between the British Paediatric Cardiac Association, representing health professionals, and the Children's Heart Federation, through recognition that each has the same objective of improving services. A notable example of this has been the joint development and authorship, along with the group known as Constructive Dialogue for Clinical Accountability, which also emerged in the aftermath of Bristol, of a guide to standards of care in the paediatric cardiac services. ${ }^{6}$ This document has had a wide circulation within the United Kingdom to hospital and primary care trusts, paediatric cardiac units, and parents. It also strongly influenced the thinking and proposals of the review group delegated by the Department of Health to consider national standards. It clearly carried weight because of its development and endorsement by both providers and users of the service.

A recently published study ${ }^{7}$ on the changing relationships of health professionals and patients considers that, while there is much discussion and debate about the involvement of patients, and a move towards services centred upon patients, there is little agreement on what these terms really mean. It goes on to say that, to date, the focus has been on the involvement of the public and users at the level of setting policies, and little attention has been given to the conduct of face-to-face encounters between patients and health professionals. The authors conclude that, while there is evidence of some professional leaders showing "enthusiasm, self-reflection and innovation" for the changes, "the continuing grip of central government and professional groups on services, however, cast doubts on the extent to which power and control will in reality be shifted to patients".

From my experience, I have some sympathy with this view. As representatives of parents on a number of bodies seeking to implement the recommendations of the various reports, my colleagues and I have been welcomed and listened to with courtesy and attention by policy makers and health professionals. Indeed many of the changes we have advocated have been incorporated into proposals and standards. But concessions at the level of deciding policy are, in 
fact, much less threatening to the professional culture, as they can subsequently be mediated through "the system" before affecting the sharp end on the ward and in the consulting room. In some more local settings, noticeable is the continuance of an "insideout" approach, where a "patient-centred service" is seen as bolting on to the current systems and culture a process of consultation with patients. In this model, reports, standards, and policies continue to be developed, articulated and negotiated within the organisation and its culture, and produced in the language of that culture. It is only when the policymakers, managers, and professionals have reached their accommodations that representatives of parents are brought into consultation. This is keeping the patient as "tail end Charlie", rather than at the heart of the process. I agree with the recommendation of this study that, to help health professionals design, plan and deliver patient-centred care, there is a need for wider research that draws on, and explains, face-to-face relationships between professionals and patients. We are very keen to see this come about. The extent to which it is accepted, or barriers erected, will give some indication of the real willingness to embrace change.

Seeking and giving consent is one of the most important elements of this relationship. This process has been the subject of considerable attention in the United Kingdom subsequent to the events at Bristol, at both national and unit levels. As a result, there is now national guidance for patients, carers, and parents to assist their understanding and full participation in the process. A national consent form provides the framework for clinician and patient to discuss options for treatment and potential benefits and consequences, all with the aim of ensuring "informed consent".

It is thus interesting to consider the study ${ }^{8}$ from the United States of America seeking to gain a better understanding of parental choice for one or two stage complete repair in paediatric cardiac surgery, where these are genuine options. The interaction of the options can be puzzling for parents, and thus achieving informed consent is itself more complex. The authors of the study ${ }^{8}$ recognise its limitations. Its subjects were parents not under real life pressures, but with those who had already been told that, in fact, their child had a normal heart. The potential for misunderstanding of risk and probability by parents is also recognised. We receive regular calls on the helpline run by the Children's Heart Federation from parents who remain unsure about these concepts. No checks appear to have been made in this study to confirm parental understanding. It is also not clear whether participants were told of potential non-fatal complications of surgery. This could be a significant factor in choices between one or two stage repair. These factors may have militated against these parents giving a full informed consent. The two major cultural differences, of the majority of the population being Spanish-speaking and the influence of health insurance, further limits its applicability to countries other than the United States of America. This is, nonetheless, an interesting study of a largely uncovered area. It would be valuable in further work to gain insight into the reasons why parents make the choices they do.

Two further studies ${ }^{9,10}$ in this fascicle are on the views of patients and their parents on services provided for the rehabilitation they would like to see to meet their continuing needs in the community. These provide formal confirmation of a large body of anecdotal evidence derived from the helpline of the Children's Heart Federation, conference workshops, ${ }^{11}$ support groups, and the visiting team from the review group. Almost all of the comments recorded in these studies I have heard many times from parents in the past two years. While it would be an overstatement to say that paediatric cardiac services cease at the door of the paediatric cardiac unit, it sometimes feels like that to parents, despite the best efforts of overstretched and understaffed cardiologists and cardiac liaison nurses. With the lower mortality rates of recent years, and more children surviving with complex conditions, the pressures have increased in the community on many parents and families, but this is not reflected in resources allocated. Parents travelling between the tertiary, secondary, and primary sectors of care often feel that they are traversing a series of rift valleys, in which there are few bridges, and even less bridge builders to smooth their journey. Parents do not have the feeling that they are being supported by a "joined-up" service with consistent standards and clear lines of communication. Units are well aware of these deficiencies, and interesting approaches are being trialed in several centres, but underfunding is hampering development. The Children's Heart Federation is pressing for increased resources for local services, and are strongly of the view that a significant proportion of any extra funds becoming available should be directed towards more cardiologists, specialist paediatricians, cardiac liaison nurses and community services to address these issues. These two studies will add further weight to our campaign.

\section{References}

1. The National Health Service Plan. A plan for investment. A plan for reform. Department of Health 2000.

2. Kennedy I. The Report of the Public Inquiry into children's heart surgery at the Bristol Royal Infirmary 1984-1995. CM 5207(1) 2001. 
3. Royal Liverpool Children's Inquiry: report. Department of Health 2001.

4. Evans R. The Report of the Independent Inquiries into Paediatric Cardiac Services at the Royal Brompton Hospital and Harefield Hospital. Royal Brompton and Harefield NHS Trust 2001.

5. Learning from Bristol. The Department of Health's Response to the Report of the Public Inquiry into children's heart surgery at the Bristol Royal Infirmary 1984-1995. Department of Health 2002.

6. Children's Heart Services - a guide to care standards. Children's Heart Federation 2001.

7. Gillespie R, Florin D, Gillam S. Changing relationships: findings from the patient involvement project. King's Fund 2002.
8. Chang RKR, Joyce JJ. Parental preference for one-stage versus two-stage surgical repair for children with congenital heart disease. Cardiol Young 2003; 13: 7-10.

9. Kendall L, Sloper P, Lewin RJP, Parsons JM. The views of young people with congenital cardiac disease on designing the services for their treatment. Cardiol Young 2003; 13: 11-19.

10. Kendall L, Sloper P, Lewin RJP, Parsons JM. The views of parents concerning the planning of services for rehabilitation of families of children with congenital cardiac disease: parents' views. Cardiol Young 2003; 13: 20-27.

11. Expectation for the Future - Our Collective Voice - Report of the parents' conference into children's heart services. Children's Heart Federation 2001. 